Supplementary Information for:

\title{
Mie-Resonant Three-Dimensional Metacrystals
}

Seokhyoung Kim, ${ }^{1,2 \dagger}$ Cindy Y. Zheng, ${ }^{1,2 \dagger}$ George C. Schatz, ${ }^{1,2}$ Koray Aydin, ${ }^{2,3}$ Kyoung-Ho Kim, ${ }^{4 *}$ Chad A. Mirkin ${ }^{1,2 *}$

${ }^{1}$ Department of Chemistry, Northwestern University, Evanston, IL 60208, USA

${ }^{2}$ International Institute for Nanotechnology, Northwestern University, Evanston, IL 60208, USA

${ }^{3}$ Department of Electrical and Computer Engineering, Northwestern University, Evanston, IL 60208, USA

${ }^{4}$ Department of Physics, Chungbuk National University, Cheongju 28644, Republic of Korea

†These authors contributed equally

*Correspondence should be addressed to Chad A. Mirkin: chadnano@northwestern.edu (for experimental work) or Kyoung-ho Kim: kyoungho@chungbuk.ac.kr (for theory). 


\section{Table of Contents}

1. Materials and Methods

1-1. Nanocube synthesis and characterization

STEM images of synthesized Au nanocube

Supplementary Figure 2. Distribution of edge lengths measured from 200 nanocubes

1-2. DNA synthesis, purification, and design

Supplementary Table 1. DNA sequences used in this work

1-3. Nanocube functionalization and assembly

1-4. Silica embedding of SLs

1-5. SL Characterization

Supplementary Figure 3. SEM images of SLs

1-6. Removal of excess silica

1-7. Sample preparation for synchrotron measurements

1-8. Finite-Element Modeling

1-9. Synchrotron Extinction Measurements

2. Determination of complex effective parameters of Au nanocube SLs

Supplementary Figure 4. Effective optical constants of nanocube SL slabs of varying thicknesses

3. Additional Supplementary Figures

Supplementary Figure 5. Electric field enhancement patterns of $\mathrm{TE}_{01}$ and $\mathrm{TE}_{11}$ modes

Supplementary Figure 6. Field patterns MD and ED resonances in 3D metacrystals

Supplementary Figure 7. Effect of various defects

Supplementary Figure 8. Size Analysis of SLs used for measurements shown in Fig. 4.

Supplementary Figure 9. Reflection and transmission spectra of the array of metacrystals 


\section{Materials and Methods}

\section{1-1. Nanocube synthesis and characterization}

All precursors were purchased from Sigma-Aldrich and used without further purification. Uniform Au nanocubes were synthesized using a seed-mediated method developed by O'Brien et $a l .{ }^{1}$ which yields $>95 \%$ of the desired shape with $<5 \%$ variation in size. In brief, single-crystalline spherical Au nanoparticles were synthesized and chemically refined through iterative reductive growth and oxidative dissolution reactions leading to uniform spherical nanoparticles, which were subsequently used as seeds to template the growth of anisotropic nanoparticles, including these nanocubes.

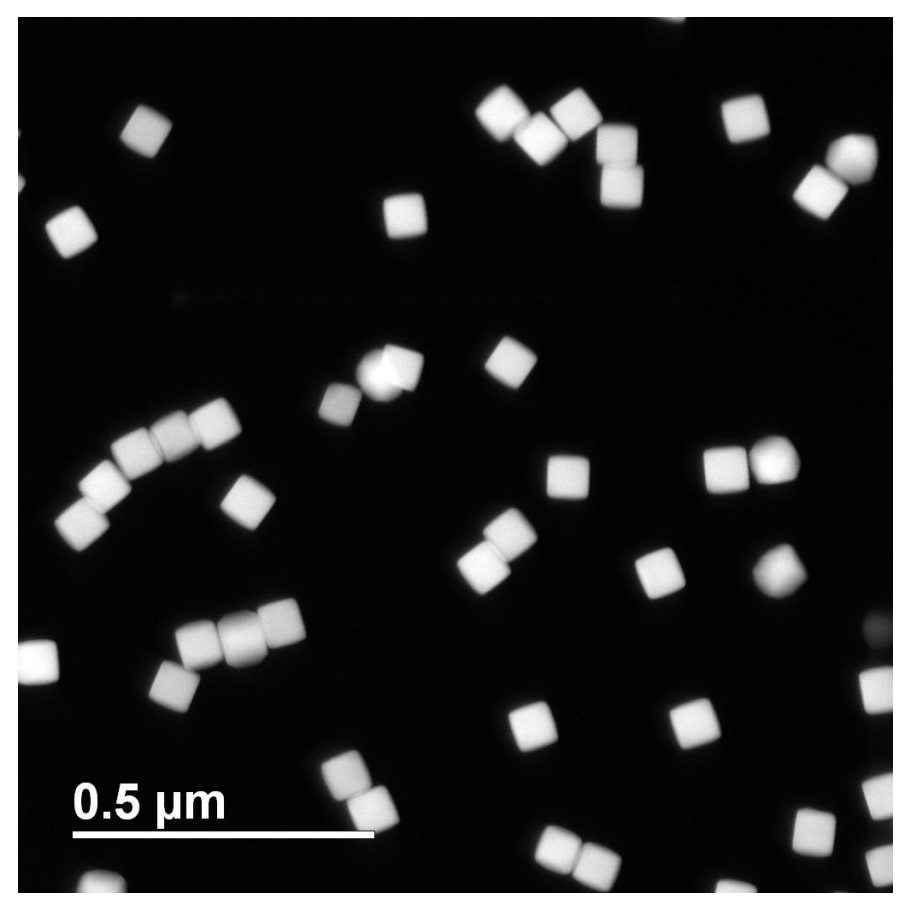

Supplementary Figure 1 | STEM image of Au nanocubes with an average edge length of $67 \pm 2$ nm. 


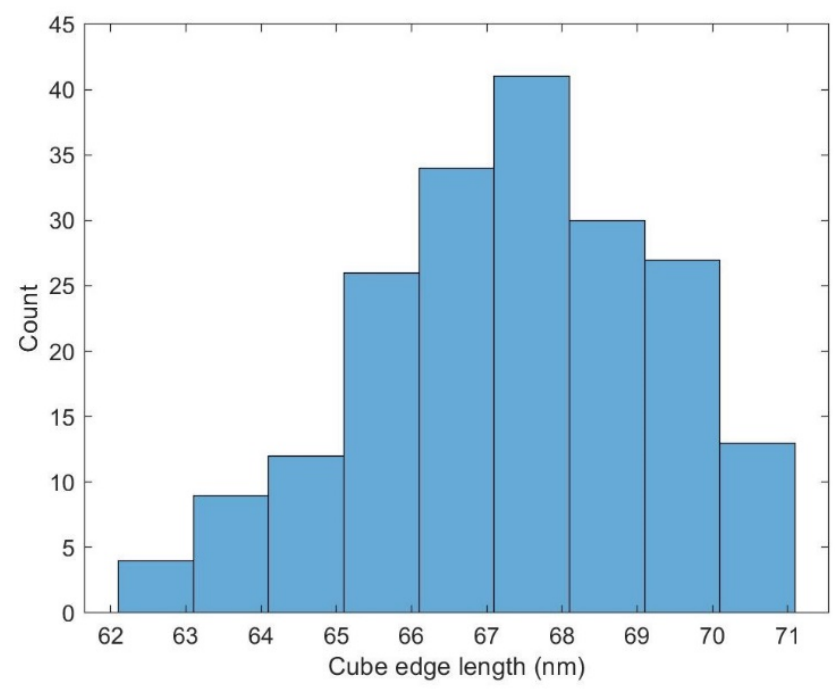

Supplementary Figure 2 | Distribution of edge lengths measured from 200 nanocubes.

Nanocubes were imaged on a Hitachi HD2300 STEM with an accelerating voltage of 200 $\mathrm{kV}$ in Z-contrast mode and the edge lengths of 200 nanocubes were measured using ImageJ. The average edge length of the cubes was determined to be $67 \pm 2 \mathrm{~nm}$.

\section{1-2. DNA synthesis, purification, and design}

All oligonucleotides used in this work were synthesized on a BioAutomation MerMade 12 (MM12) automated oligonucleotide synthesizer using reagents purchased from Glen Research. After synthesis, all DNA was purified with reverse-phase high-performance liquid chromatography (HPLC, Agilent) followed by standard deprotection procedures. Matrix-assisted laser desorption ionization time-of-flight mass spectrometry (MALDI-TOF-MS) was used to characterize the purified oligonucleotides and confirm their molecular weight. 
The DNA sequences used in this work are listed in Supplementary Table 1 and are taken from the literature. ${ }^{2}$ Black denotes spacer regions, purple denotes thiols, blue denotes complementary regions in the anchor and linker strands, and red denotes the self-complementary sticky end binding region. Sp18 is a hexaethylene glycol spacer unit used to increase flexibility of the DNA. The sequences were modified such that the single-stranded spacer regions in both the anchor and linker were replaced with more flexible sp18 spacer units. The extra flexibility was needed so that large nanocubes functionalized with short DNA ligands could form well-defined single crystals instead of the amorphous, kinetic structures that otherwise form.

Supplementary Table 1. DNA sequences used in this work

\begin{tabular}{|l|l|}
\hline \multicolumn{1}{|c|}{ Name } & \multicolumn{1}{c|}{ Sequence (5' to 3') } \\
\hline Anchor & TCA ACT ATT CCT ACC TAC (sp18)2 SH \\
\hline Self-Complementary Linker & GTA GGT AGG AAT AGT TGA (sp18) GCGC \\
\hline $\begin{array}{l}\text { Self-Complementary Linker } \\
\text { for Control in Fig. S3 }\end{array}$ & GTA GGT AGG AAT AGT TGA A GCGC \\
\hline
\end{tabular}

\section{1-3. Nanocube functionalization and assembly}

Nanocubes were functionalized using a procedure modified from that described by Jones et $a l .^{3}$ and O'Brien et $a .^{2}$ Briefly, 3' propyl-modified oligonucleotides were first treated with a $100 \mathrm{mM}$ solution of dithiotreitol (DTT) in $170 \mathrm{mM}$ sodium phosphate buffer $(\mathrm{pH}=7.4)$, followed by purification on a Nap-5 size exclusion column (GE Life Sciences) to remove excess DTT. During this time, $1 \mathrm{~mL}$ aliquots of the as-synthesized cube solutions ( $\sim 1$ OD) were centrifuged for $8 \mathrm{~min}$ at 5,800 rpm, the supernatant was removed, and the nanoparticles were resuspended in 
water. The cubes were then centrifuged a second time for $8 \mathrm{~min}$ and 5,800 rpm, the supernatant was removed, and then purified DNA was added to the pellet. 1 OD of DNA was added per each $1 \mathrm{~mL}$ of particles. The particle solution was then brought to $0.01 \mathrm{M}$ sodium phosphate buffer $(\mathrm{pH}$ $=7.4$ ) and 0.01 wt. $\%$ sodium dodecyl sulfate (SDS) in water. Stepwise addition of $2 \mathrm{M} \mathrm{NaCl}$ was carried out every half hour, such that the $\mathrm{NaCl}$ concentration was increased to $0.05 \mathrm{M}, 0.1 \mathrm{M}, 0.2$ $\mathrm{M}, 0.3 \mathrm{M}, 0.5 \mathrm{M}$ respectively. Following this process, the nanoparticles were placed on a shaker at $1,000 \mathrm{rpm}$ and left overnight to ensure a dense loading of oligonucleotides. After functionalization, the nanoparticle solutions were centrifuged three times to remove excess DNA. After each of the first two rounds of centrifugation, the nanoparticles were resuspended in 0.01 wt. \% SDS, and after the last centrifugation step, the particles were resuspended in $0.3 \mathrm{M} \mathrm{NaCl}$, $0.01 \mathrm{M}$ sodium phosphate buffer $(\mathrm{pH}=7.4)$, and $0.01 \mathrm{wt}$ \% SDS. To the nanoparticle solution, 25,000 linker strands were added per nanoparticle (in excess of the number of thiolated strands). Nanoparticle solutions were prepared such that the final concentration was 8 OD in a total volume of $100 \mu \mathrm{L}$. Each solution was then pipetted into $200-\mu \mathrm{L}$ PCR eight-tube strips (Life Technologies) and placed into a thermal cycler (Life Technologies). The temperature of the thermal cycler was slowly cooled from $70{ }^{\circ} \mathrm{C}$ (where the particles are fully discrete) to $20{ }^{\circ} \mathrm{C}$ (where the particles are fully associated) for all samples at a rate of $0.1{ }^{\circ} \mathrm{C} / 10 \mathrm{~min}$. The slow speed of this temperature change ensures that the system has enough time to reach equilibrium at each temperature.

\section{1-4. Silica embedding of SLs}

Nanocube SLs were encapsulated in silica according to literature precedent. ${ }^{4}$ This method largely preserves the crystal symmetry and lattice parameters of the lattice in the solid state. Slowcooled samples were transferred to a $1.5 \mathrm{~mL}$ Eppendorf tube, and the volume was brought up to 1 
$\mathrm{mL}$ with an overall $\mathrm{NaCl}$ concentration of $0.5 \mathrm{M}$. To this solution, $2 \mu \mathrm{L}$ of the quaternary silane salt, N, trimethoxysilylpropyl-N,N,N-trimethylammonium chloride (TMSPA), was added and the tube was mixed at room temperature on a thermomixer (Eppendorf) at a rate of $700 \mathrm{rpm}$ for 30 min. Then, $4 \mu \mathrm{L}$ of triethoxysilane (TES) was added to the solution and allowed to form a silica network around the entire lattice. The mixture was left on the thermomixer at $700 \mathrm{rpm}$ for four days, followed by three rounds of centrifugation, removal of supernatant, and resuspension in Nanopure water. Note, in the case of successful silica embedding, the addition of water does not result in lattice dissolution (i.e., the solution does not turn pink).

\section{1-5. SL characterization}

SLs were imaged on a Hitachi SEM SU8030 instrument at an accelerating voltage of 5 or $30 \mathrm{kV}$. Silica-embedded SLs were redispersed in $100 \mu \mathrm{L}$ of ethanol and the solution was vortexed to break up aggregates. Next a $5 \mu \mathrm{L}$ droplet of the solution was drop-cast onto a small piece of silicon wafer.

SLs assembled with a sp18 unit in the linker formed crystals with larger domain sizes, and a higher proportion of well-formed crystal habits (Supplementary Fig. 3a,c,d) compared to those assembled without one (Supplementary Fig. 3b). High-magnification images were taken of the SLs surfaces using an accelerating voltage of $30 \mathrm{kV}$ (Supplementary Fig. 3e) to quantify the periodicity of the SLs and deduce their gap distance by subtracting the average cube size measured from STEM images $(67 \mathrm{~nm})$. Periodicity was measured using ImageJ. Specifically, the distance across 3 periods was measured for higher precision, and then this value was divided by 3 to obtain the periodicity. This measurement was done for 50 different regions and averaged. The average periodicity was determined to be $73 \mathrm{~nm}$ giving an average gap of $6 \mathrm{~nm}$. 

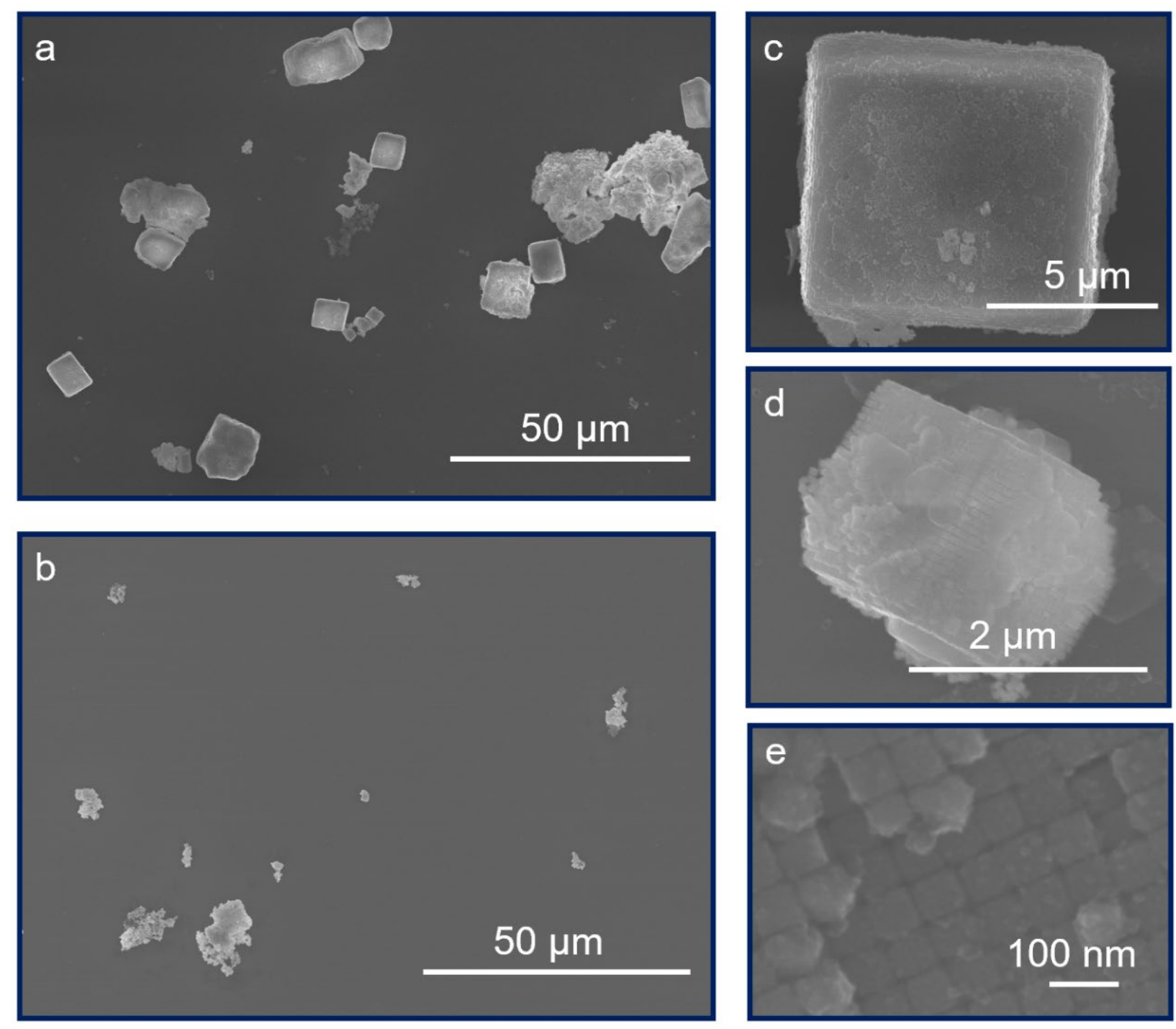

Supplementary Figure 3 | a, Large-area SEM images showing SLs formed with our modified DNA design. b, SEM image of SLs formed with the same DNA design except with the sp18 unit in the linker replaced by a single-stranded A base. $\mathbf{c}, \mathbf{d}$, Magnified SEM images of representative SLs in a. e, High-magnification SEM image of a SL surface used to quantify gap size. 


\section{1-6. Removal of excess silica}

Because silica exhibits strong absorption in the mid-IR region, excess silica formed during the embedding process had to be removed before optical characterization. To do this, a density gradient separation method was employed. $50 \mu \mathrm{L}$ of sodium metatungstate solution was pipetted to the bottom of a $1.5 \mathrm{~mL}$ Eppendorf tube and $30 \mu \mathrm{L}$ of the silica embedded SLs in water was added to the top of the sodium metatungstate solution, forming two layers. After $12 \mathrm{~h}$, all the SLs fell into the bottom layer because of their heavier density, while the excess silica remained in the top layer. The top layer was carefully removed with a pipette. To remove the sodium metatungstate, the SLs were washed $5 \mathrm{x}$ with water, centrifuging each time at $12,000 \mathrm{rpm}$ for $10 \mathrm{~s}$ and subsequently removing the supernatant. The SLs were redispersed in $100 \mu \mathrm{L}$ of water.

\section{1-7. Sample preparation for synchrotron measurements}

Double-side-polished silicon wafers were cut into $\sim 1.5 \times 1.5 \mathrm{~cm}^{2}$ pieces and cleaned by sonicating in acetone and IPA and then blown dry using $\mathrm{N}_{2}$. Next, the wafers were plasma cleaned with an $\mathrm{O}_{2}$ plasma at $50 \mathrm{~W}$ for 5 min. Immediately following plasma cleaning, $10 \mu \mathrm{L}$ of the SL solutions were dropped onto the wafer and the wafer was placed in a desiccator to dry. Isolated SLs of desired sizes for synchrotron FTIR measurements were found using SEM and their positions on the substrate were tracked with a series of SEM images at different magnifications and corresponding optical microscopy images.

1-8. Finite-Element Modeling. Numerical modeling was performed using COMSOL Multiphysics with the wave optics module. The effective parameters were extracted by a modified NicholsonRoss-Weir (NRW) method from full-field calculations using infinite slabs of SLs. A refractive 
index of 1.5 was used for silica and DNA. Scattering calculations in 2D and 3D geometries were performed with SL structures under a plane wave surrounded by a large air domain terminated by perfectly matched layers. The calculation for Fig. 5 was performed using an effective crystal with optical constants derived from Fig. $2 b$ with an in-plane periodic boundary condition. The mesh size in the Au bars and gap region was set to no larger than the gap distance, and a predefined extra fine mesh size was used for the rest of the simulation region. The calculations were done in the frequency domain using a direct solver. The scattering and absorption efficiencies were calculated by integrating relative normal Poynting vectors around the SL boundaries (scattering) and power sloss in the SL volume (absorption) normalized by the illumination power density and physical cross-section of the SLs.

1-9. Synchrotron Extinction Measurements. SL samples were dispersed in water and dropcasted on double-side-polished $<100>$ silicon substrates (University Wafer) and dried under vacuum. The samples were placed on an FTIR microscope equipped with a Mercury-CadmiumTelluride (MCT) detector (Nicolet, Thermo Fisher) in the far-field infrared beamline (1.4) at the Advanced Light Source, National Lawrence Berkeley Laboratory. Synchrotron infrared radiation was directed through a Cassegrain objective lens with $36 \times$ magnification and focused into diffraction limited spot sizes. Blank measurements were collected from clean regions of the $\mathrm{Si}$ substrate, and the sample measurements were collected with the beam focused on the SLs of interest. Transmission $(T)$ was measured by averaging 256 scans for each sample, and extinction was calculated by $1-T$. 


\section{Determination of complex effective parameters of Au nanocube SLs}

The real and imaginary parts of effective permittivity and permeability were calculated with a modified Nicholson-Ross-Weir (NRW) method ${ }^{5,6}$ and extrapolated to the bulk limits. ${ }^{7}$ First, we consider infinite 2D slabs of Au nanocube SLs with $a=60 \mathrm{~nm}, g=2 \mathrm{~nm}$, and thickness varying from 1 to 5 layers. With the S-parameters obtained from FEM, we retrieve the real and imaginary parts of the permittivity (Supplementary Figs. 4a-b) and permeability (Supplementary Figs. 4c-d).

We only show values for wavelengths longer than the positions of the first $\mathrm{FP}$ modes $\left(\mathrm{FP}_{0}\right)$ to eliminate artifacts associated with a phase-inversion (see Supplementary Fig. 4e for the phasecorrected permittivity curve for 5 layers). The plots in Supplementary Fig. 4a-d show that all curves converge to single values, indicating that the optical constants away from the FP modes do not depend on the number of layers. Upon closer inspection of the tail regions (insets of Supplementary Figs. 4a and c), we find that the asymptote values slightly decrease as more layers are added but approach the bulk limits obtained by analytical modeling. We thus adopt these bulk values in all subsequent analyses. For the imaginary parts, we estimate the bulk limits by extrapolating to infinity the values obtained for several finite thicknesses, using the multiplication factor obtained from the real part. Specifically,

$$
\operatorname{imag}(\infty)=\operatorname{imag}(2)-[\operatorname{imag}(2)-\operatorname{imag}(5)] * \frac{\operatorname{real}(2)-\operatorname{real}(\infty)}{\operatorname{real}(2)-\operatorname{real}(5)}
$$

where imag $(\mathrm{m})$ and real $(\mathrm{m})$ are the imaginary and real parts of the optical constants, respectively, for an m layer slab of Au nanocubes.

This calculation was repeated for $g$ values from 2 to $10 \mathrm{~nm}$, and the long-wavelength values of the real and imaginary parts of effective parameters obtained at $12.4 \mu \mathrm{m}$ are plotted in Fig. $2 \mathrm{~b}$ of the main text. We assume that the effective parameters are wavelength-independent in the 2-15 
$\mu \mathrm{m}$ wavelength range because this is within a non-dispersive quasi-static regime for Au nanocubes of the sizes used in this study.

a

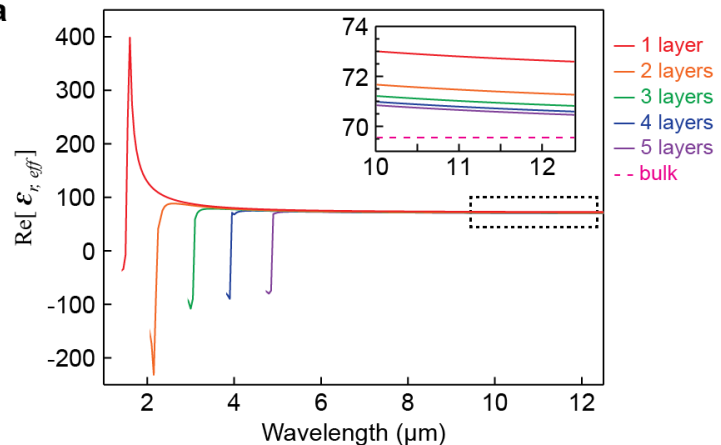

c
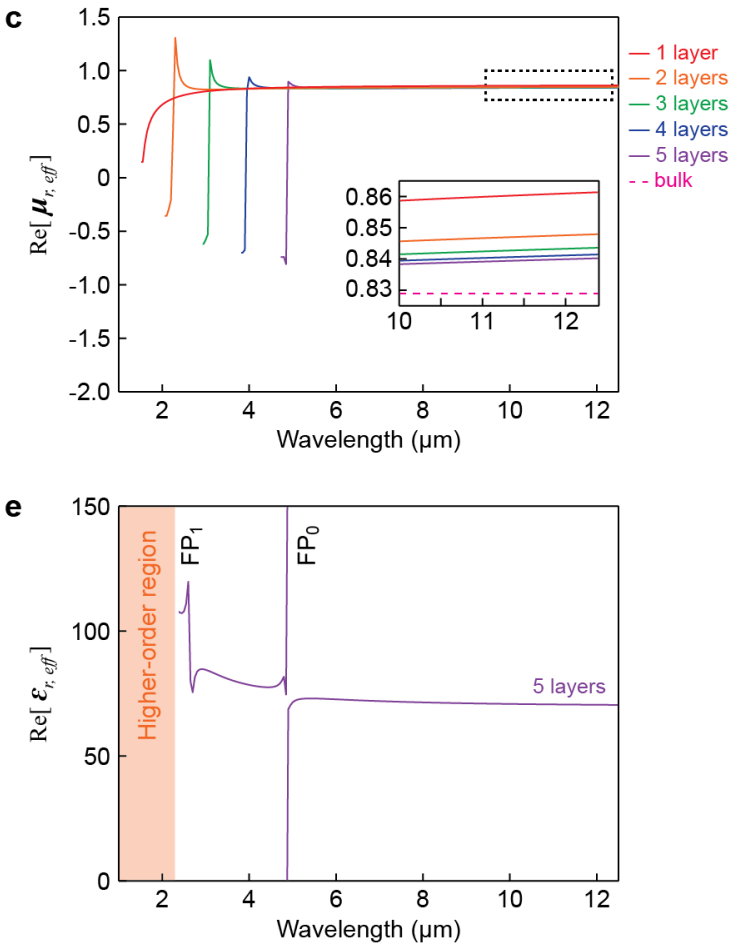

b

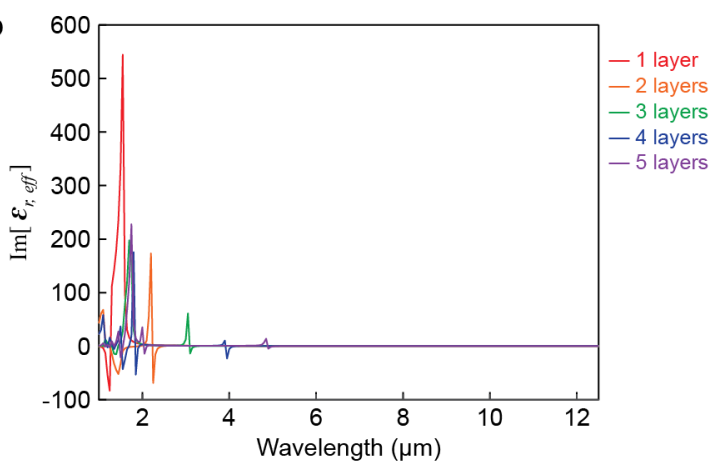

d

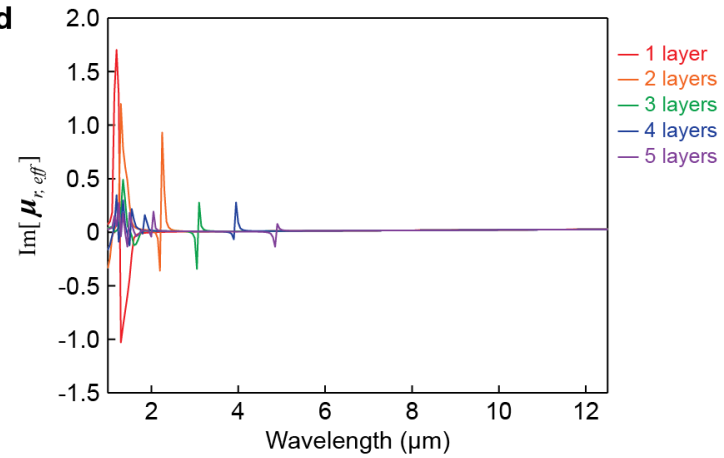

Supplementary Figure $4 \mid$ Effective optical constants of nanocube SL slabs of varying thicknesses. a-d, Real (a, c) and imaginary $(\mathbf{b}, \mathbf{d})$ parts of the retrieved effective permittivity (a, b) and permeability $(\mathbf{c}, \mathbf{d})$ of nanocube SL slabs with $a=60 \mathrm{~nm}, g=2 \mathrm{~nm}$, and varying number of layers from 1 to 5. Insets in panels a and c are magnified views of the boxed regions. Bulk values in the insets are from an analytical model. ${ }^{7} \mathbf{e}$, Real part of the effective permittivity for 5 layers with phase corrected before and after the first $\mathrm{FP}$ mode $\left(\mathrm{FP}_{0}\right)$. 
a

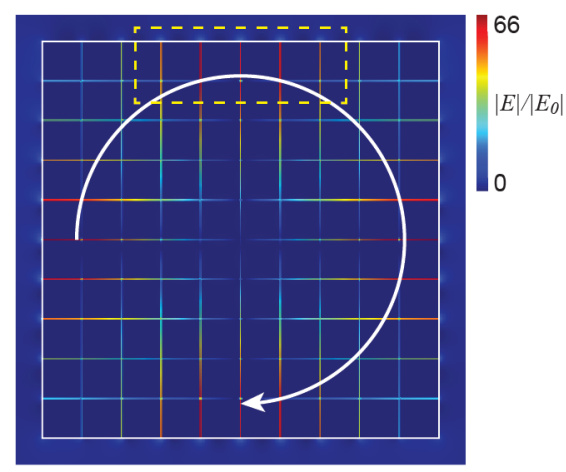

b

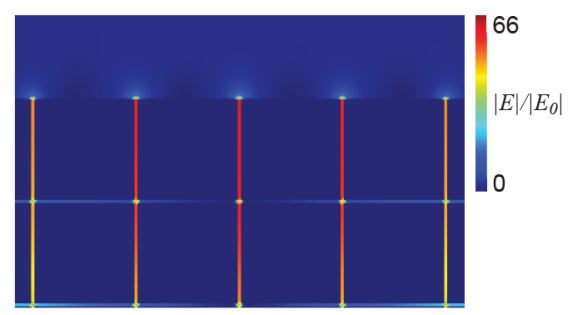

C

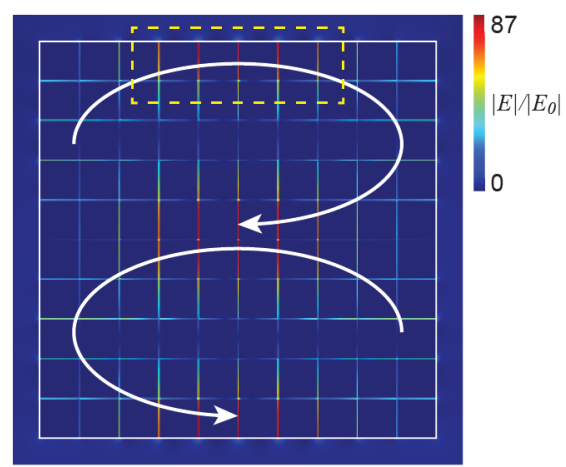

d

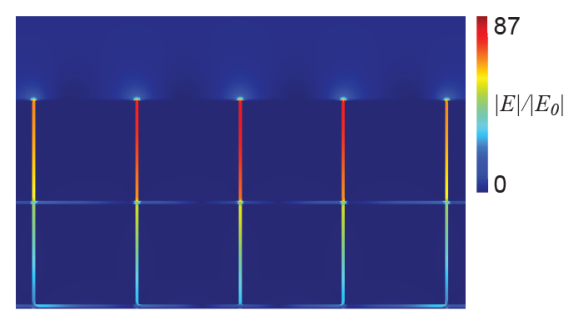

Supplementary Figure 5 | Electric field enhancement patterns of TE 01 (a-b) and $\mathrm{TE}_{11}(\mathbf{c}-\mathbf{d})$ modes from Fig. 2f-g. Panels $\mathbf{b}$ and $\mathbf{d}$ are magnified views of the boxed regions in panels $\mathbf{a}$ and $\mathbf{c}$, respectively. 
a

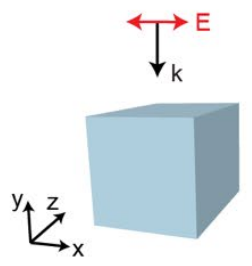

b

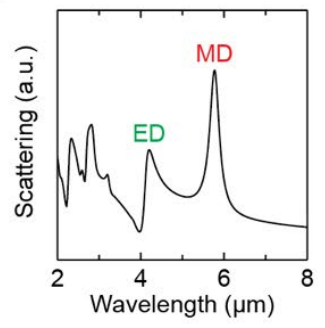

MD

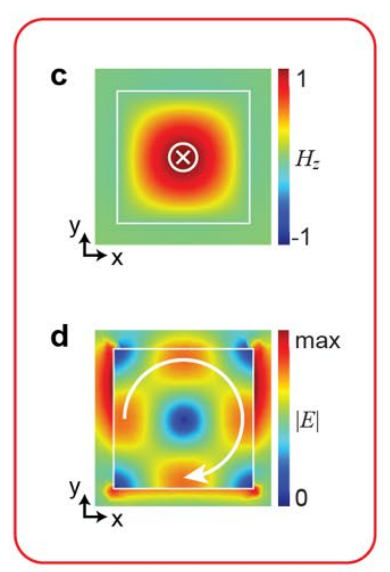

ED

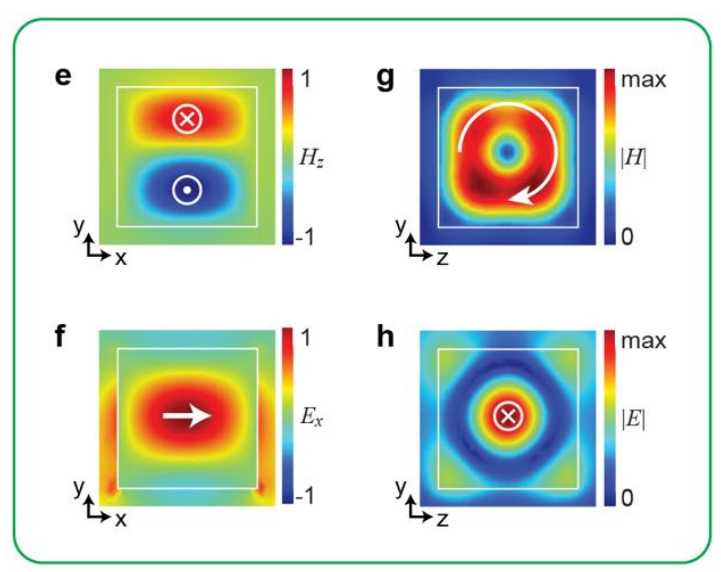

Supplementary Figure 6 | Field patterns of the MD and ED resonances in 3D metacrystals. a, Illumination geometry for a metacrystal. b, Calculated scattering spectrum of an effective metacrystal corresponding to $a=60 \mathrm{~nm}, g=2 \mathrm{~nm}, N_{x}=N_{y}=N_{z}=10$. c-h, Magnetic (top) and electric (bottom) field patterns of MD (c-d) and ED (e-h) for different plane projections. 
a
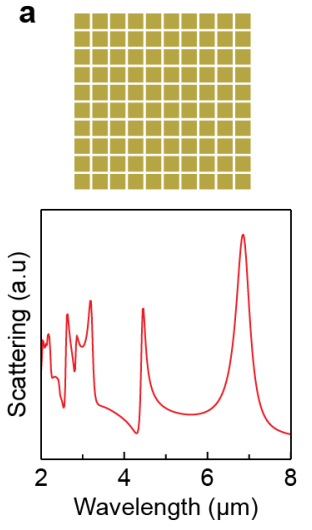

b
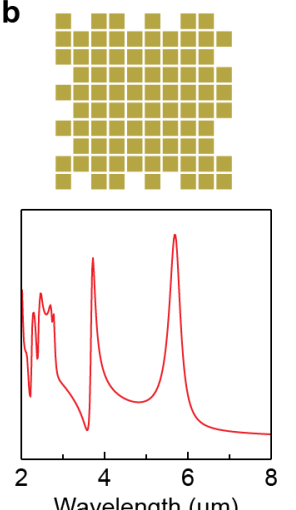

c
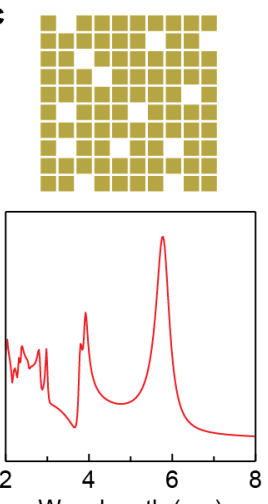

d
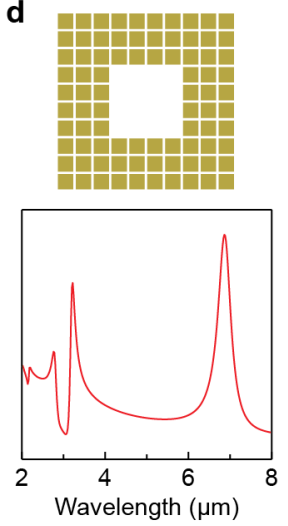

e
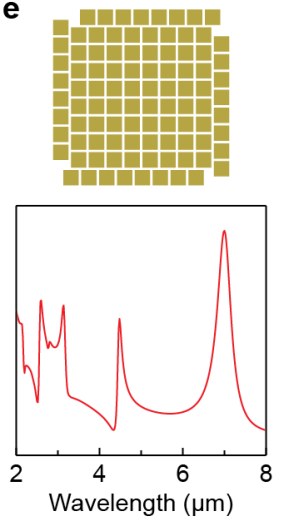

Supplementary Figure 7 | Effect of various defects. Simulation geometries (top) and calculated scattering spectra (bottom) of metacrystals with $a=60 \mathrm{~nm}, g=2 \mathrm{~nm}$, and $N=10$ with no defects (a), missing particles on the surface (b), random vacancies (c), a hollow core (d), and a misaligned outermost shell (e). The main spectral features are preserved in all cases, albeit with minor shifts in peak positions. 


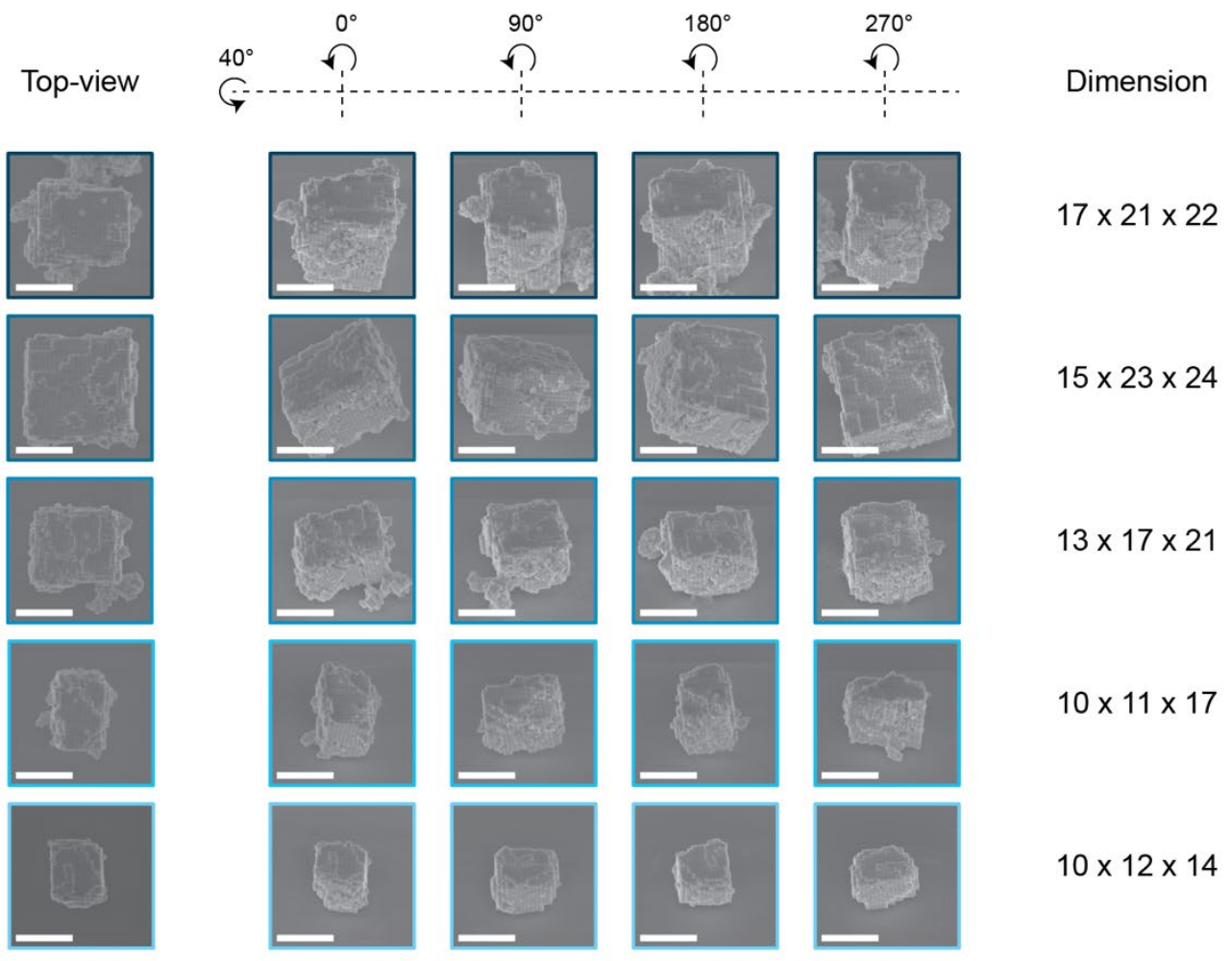

Supplementary Figure $8 \mid$ Size analysis of SLs used for measurements shown in Fig. 4. Top-view SEM images (left), tilted images at a $40^{\circ}$ angle and various azimuthal angles (middle), and dimensions $(\mathrm{x} \times \mathrm{y} \times \mathrm{z})$ of each SL in terms of the number of nanocubes (right). All scale bars, 1 $\mu \mathrm{m}$. 


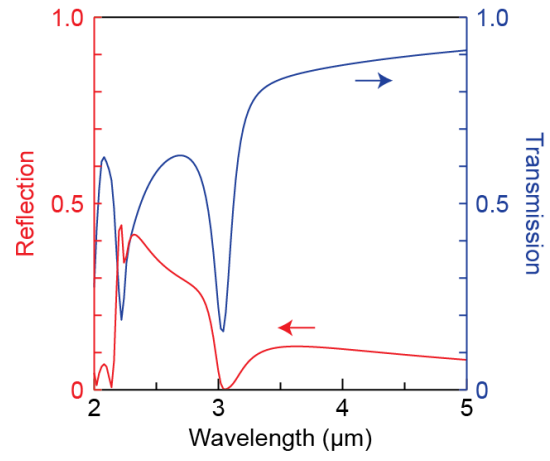

Supplementary Figure $9 \mid$ Reflection and transmission spectra of the array of metacrystals shown in Fig. 5. 


\section{References}

(1) O’Brien, M. N.; Jones, M. R.; Brown, K. A.; Mirkin, C. A. Universal Noble Metal Nanoparticle Seeds Realized through Iterative Reductive Growth and Oxidative Dissolution Reactions. J. Am. Chem. Soc. 2014, 136, 7603-7606.

(2) O’Brien, M. N.; Lin, H. X.; Girard, M.; Olvera De La Cruz, M.; Mirkin, C. A.

Programming Colloidal Crystal Habit with Anisotropic Nanoparticle Building Blocks and DNA Bonds. J. Am. Chem. Soc. 2016, 138, 14562-14565.

(3) Jones, M. R.; Macfarlane, R. J.; Lee, B.; Zhang, J.; Young, K. L.; Senise, A. J.; Mirkin, C. A. DNA-Nanoparticle Superlattices Formed from Anisotropic Building Blocks. Nat. Mater. 2010, 9, 913-917.

(4) Auyueng, E.; MacFarlane, R. J.; Choi, C. J. J.; Cutler, J. I.; Mirkin, C. A. Transitioning DNA-Engineered Nanoparticle Superlattices from Solution to the Solid State. Adv. Mater. 2012, 24, 5181-5186.

(5) Paula, A. L. de; Rezende, M. C.; Barroso, J. J. Modified Nicolson-Ross-Weir (NRW) Method to Retrieve the Constitutive Parameters of Low-Loss Materials. In 2011 SBMO/IEEE MTT-S International Microwave and Optoelectronics Conference (IMOC 2011); 2011; pp 488-492.

(6) Nicolson, A. M.; Ross, G. F. Measurement of the Intrinsic Properties of Materials by Time-Domain Techniques. IEEE Trans. Instrum. Meas. 1970, 19, 377-382.

(7) Chung, K.; Kim, R.; Chang, T.; Shin, J. Optical Effective Media with Independent Control of Permittivity and Permeability Based on Conductive Particles. Appl. Phys. Lett. 2016, $109,21114$. 